\title{
L'ERC (European Research Council) Une chance et un défi pour les chercheurs et les organismes de recherche
}

\author{
Daniel Estève (daniel.esteve@cea.fr) \\ Service de physique de l'état condensé, CEA/Saclay, 91191 Gif-sur-Yvette
}

\begin{abstract}
L'ERC est une nouvelle institution européenne,
\end{abstract} conçue et contrôlée par des chercheurs, dans le but de soutenir les meilleurs d'entre eux, en Europe quelle que soit la discipline, et sur le seul critère de l'excellence scientifique.

\section{La genèse de l'ERC}

L'ERC est issu d'une initiative prise en 2003 durant la présidence de la Commission Européenne par le Danemark, un pays convaincu de l'importance de la recherche fondamentale et de la nécessité de lui donner une dimension européenne. La Commission vit dans cette proposition le moyen de financer tous les domaines de la recherche fondamentale, alors que la recherche finalisée, plus facilement justifiable auprès des contribuables, était privilégiée jusqu'alors. De leur côté, les politiques y virent un moyen de promouvoir le concept de «société de la connaissance ", basé sur le triangle éducation-rechercheinnovation. Ce nouveau paradigme leur semble être aujourd'hui la voie de salut pour assurer l'avenir de notre « vieille " Europe.

En 2005, un comité indépendant nomma un conseil scientifique de 22 membres pour décider de la stratégie et du mode de fonctionnement du futur European Research Council (ERC). Ce conseil scientifique, présidé par le Professeur Fotis Kafatos (Imperial College, Londres), commença à élaborer le programme de travail de l'ERC avant même son existence légale, puisque le $7^{\mathrm{e}}$ programme cadre pour la recherche et le développement ( $7^{\mathrm{e}}$ PCRD) ne fut adopté qu'à l'automne 2006. Le programme de travail proposé est actuellement mis en œuvre par une structure dédiée dépendant de la Commission, mais dont le contrôle reste aux mains des scientifiques. L'adoption du budget européen de la recherche à l'automne 2006 a fixé le budget de l'ERC à 7,5 milliards d'Euros pour l'ensemble du 7 PCRD (début 2007 à fin 2013), ce qui correspond à environ 15\% des moyens attribués à la recherche.

\section{La stratégie de l'ERC : deux types d'appels à projets}

Vu ce budget limité et le nombre de domaines de recherche concernés (tous), l'ERC a décidé de se focaliser sur deux types d'actions :

- les starting grants, pour soutenir les jeunes chercheurs quand ils prennent la responsabilité d'une équipe ou d'un programme de recherche ;
- les established grants, pour soutenir les meilleurs chercheurs à tout stade de leur carrière.

Ces deux types d'actions partagent le même objectif : soutenir les chercheurs ayant auparavant le mieux démontré leur aptitude à effectuer des recherches de qualité et proposant des projets innovants, à fort impact potentiel sur l'avancée des connaissances. L'évaluation des dossiers se fera donc au niveau du porteur et au niveau du projet, avec des poids appropriés. Les bourses ERC seront d'un montant élevé, pouvant atteindre $2 \mathrm{M} €$ pour les starting grants et 2,5 M€ pour les established grants, sur une durée de 5 ans. L'emploi des fonds sera d'une grande flexibilité : personnel, équipement..

Un autre point important est la simplification des procédures de contrôle. Un rapport annuel léger, une revue de projet à mi-parcours avec éventuellement des visites sur site, et un rapport final en seront les éléments principaux. Les milestones $^{1}$ et deliverables ${ }^{2}$, concepts peu pertinents pour la recherche fondamentale, n'auront pas cours à l'ERC. L'utilisation des fonds fera l'objet d'un rapport financier, selon les règles du $7^{\mathrm{e}} \mathrm{PCRD}$.

\section{Des comités thématiques \\ favorisant les projets \\ pluridisciplinaires}

L'évaluation des propositions sera effectuée par des comités thématiques, regroupés en trois grands domaines : sciences humaines, sciences de la vie, autres sciences.

Ce troisième et plus vaste domaine couvre les mathématiques, la physique, la chimie, l'informatique, l'ingénierie, les sciences de l'univers et les sciences de la Terre. Le libellé précis des comités thématiques qui en dépendent se trouve dans l'encadré page 22. On constate d'emblée que les champs disciplinaires ne sont pas rangés dans des silos étanches. Ces recouvrements sont destinés à favoriser les projets à caractère pluridisciplinaire et innovant. Pour la physique, la pluridisciplinarité s'opère avec les mathématiques, l'informatique, la chimie, les sciences de l'univers et de la Terre, mais aussi avec la biologie. 


\section{L'appel starting grants}

Les règles en sont détaillées dans l'appel à propositions disponible sur le site CORDIS de la Commission Européenne. Le budget alloué à l'appel starting grants est proche de $300 \mathrm{M} €$. Les chercheurs éligibles doivent être entre deux et neuf ans après la thèse, avec des extensions possibles (enfants, service militaire...). Ils doivent être indépendants durant l'exécution du projet, c'est-à-dire assurer la direction des recherches, être responsables des étudiants en thèse et des postdoctorants, du budget, et pouvoir signer les publications en tant que responsable (dernier auteur en général). Il est clair que ces critères sont rarement satisfaits par les jeunes chercheurs recrutés par des équipes déjà bien structurées. Pour réussir à l'appel, il faudra impérativement donner aux candidats la pleine responsabilité du projet de recherche porté, et les rendre indépendants. Même si le concept anglo-saxon de Principal Investigator (PI en jargon de dossier) prime clairement sur celui d'équipe, l'équipe du PI et les collaborations qu'il saura proposer seront très importantes dans l'évaluation du dossier. Le candidat n'a pas besoin d'être déjà embauché pour postuler à l'ERC, mais doit avoir reçu le soutien d'une institution pour son projet. Son salaire pourra même être pris sur la bourse ERC, avec une majoration possible pour les pays à bas salaires.

\section{Un effet de levier sur les organismes de recherche}

Même si le budget récolté par les organismes de recherche via l'ERC restera modeste, les résultats obtenus auront un effet de levier sur eux. En effet, les chercheurs européens seront évalués dans une compétition ouverte par les mêmes comités, et composés de membres d'une stature scientifique incontestable. Leurs laboratoires et leurs institutions de recherche seront donc aussi indirectement évalués par l'ERC. Afin de mieux réussir dans cette compétition, les organismes de recherche seront poussés à proposer un meilleur environnement de recherche, avec des structures administratives plus simples et plus efficaces. Comme il reste du chemin à faire dans cette direction, l'ERC devrait contribuer à faire bouger les lignes dans la bonne direction. Notons aussi qu'un changement d'institution sera possible, car les bourses ERC pourront être transférées à une autre institution après un délai minimal de deux ans.

\section{Conclusion}

L'ERC propose un nouveau mode de financement de la recherche fondamentale, piloté par les scientifiques et basé sur des critères d'évaluation qu'ils auront eux-mêmes choisis. La Commission et les chercheurs scruteront les résultats de ses premiers appels. Vu son budget limité, l'ERC va garder durant les prochaines années un caractère très élitiste, en sélectionnant les meilleurs parmi les meilleurs. Son succès est un enjeu important pour toute la communauté scientifique, car il contribuerait à pérenniser le financement de la recherche fondamentale dans tous les domaines au niveau européen, et à pouvoir attribuer à l'avenir des bourses ERC au grand nombre de très bons chercheurs présents en Europe.

\section{LISTE DES COMTÉS THÉMATQUUES}

Mathematics, physical sciences, information and communication, engineering, universe and earth sciences

1 - MATHEMATICAL FOUNDATIONS

All areas of mathematics, pure and applied, plus mathematical aspects of theoretical computer science, and mathematical physics.

2 - FUNDAMENTAL CONSTITUENTS OF MATTER

High energy, particle, nuclear, plasma, atomic, molecular, gas, and optical physics.

3 - CONDENSED MATTER IN PHYSICS AND CHEMISTRY

Condensed matter (structure, electronic properties, fluids...), statistical physics, nanosciences, reactions.

4 - MATERIAL AND CHEMICAL SCIENCES

Material sciences, molecular architecture, chemical theory, analysis and synthesis (organic and inorganic), physical and environmental chemistry, method development.

5 - INFORMATION AND COMMUNICATION

Informatics and information systems, computer science, scientific computing, communication technology, intelligent systems.

6 - ENGINEERING SCIENCES

Electronics, product design, process design \& control, construction methods, fluid and solid mechanics, energy systems, bio-engineering.

7 - UNIVERSE SCIENCE

Astro-physics/chemistry/biology/geology; solar system; stellar, galactic and extragalactic astronomy; cosmology; space science, instrumentation.

8 - EARTH SYSTEM SCIENCE

Physical geography, geology, geophysics, meteorology, oceanography, climatology, ecology, global environmental change, biogeochemical cycles, solar planets, natural resources management. 